\title{
Spectral theory of SG pseudo-differential operators on $L^{p}\left(\mathbb{R}^{n}\right)$
}

by

\author{
Aparajita Dasgupta and M. W. Wong (Toronto)
}

\begin{abstract}
To every elliptic SG pseudo-differential operator with positive orders, we associate the minimal and maximal operators on $L^{p}\left(\mathbb{R}^{n}\right), 1<p<\infty$, and prove that they are equal. The domain of the minimal (= maximal) operator is explicitly computed in terms of a Sobolev space. We prove that an elliptic SG pseudo-differential operator is Fredholm. The essential spectra of elliptic SG pseudo-differential operators with positive orders and bounded SG pseudo-differential operators with orders 0, 0 are computed.
\end{abstract}

1. SG pseudo-differential operators. We give in this section a precise introduction to the formal properties of SG pseudo-differential operators, also known as pseudo-differential operators with symbols of global type. In [7], they are also called pseudo-differential operators with exit behavior. SG pseudo-differential operators and related topics can be found in [3], [4], [6], [10], [12] and the references therein.

Let $m_{1}, m_{2} \in(-\infty, \infty)$. Then we let $S^{m_{1}, m_{2}}$ be the set of all functions in $C^{\infty}\left(\mathbb{R}^{n} \times \mathbb{R}^{n}\right)$ such that for all multi-indices $\alpha$ and $\beta$, there exists a positive constant $C_{\alpha, \beta}$ for which

$$
\left|\left(D_{x}^{\alpha} D_{\xi}^{\beta} \sigma\right)(x, \xi)\right| \leq C_{\alpha, \beta}\langle x\rangle^{m_{2}-|\alpha|}\langle\xi\rangle^{m_{1}-|\beta|}, \quad x, \xi \in \mathbb{R}^{n},
$$

where \langle\rangle denotes the function on $\mathbb{R}^{N}$ given by

$$
\langle z\rangle=\left(1+|z|^{2}\right)^{1 / 2}, \quad z \in \mathbb{R}^{N},
$$

for every positive integer $N$. A function in $S^{m_{1}, m_{2}}$ is said to be an $S G$ symbol of orders $m_{1}, m_{2}$. It is clear that if $\sigma \in S^{m_{1}, m_{2}}$, then $\sigma \in S^{m_{1}}$, where $S^{m_{1}}$ is the class of symbols of classical pseudo-differential operators studied extensively in the book [19] by Wong.

2000 Mathematics Subject Classification: Primary 35S05, 47G30; Secondary 47A53.

Key words and phrases: pseudo-differential operators, ellipticity, minimal and maximal operators, Fredholm operators, essential spectra, indices.

This research has been supported by the Natural Sciences and Engineering Research Council of Canada. 
Let $\sigma \in S^{m_{1}, m_{2}}$. Then we define the pseudo-differential operator $T_{\sigma}$ with symbol $\sigma$ by

$$
\left(T_{\sigma} \varphi\right)(x)=(2 \pi)^{-n / 2} \int_{\mathbb{R}^{n}} e^{i x \cdot \xi} \sigma(x, \xi) \hat{\varphi}(\xi) d \xi, \quad x \in \mathbb{R}^{n},
$$

for all functions $\varphi$ in the Schwartz space $\mathcal{S}$, where

$$
\hat{\varphi}(\xi)=(2 \pi)^{-n / 2} \int_{\mathbb{R}^{n}} e^{-i x \cdot \xi} \varphi(x) d x, \quad \xi \in \mathbb{R}^{n} .
$$

It can be proved easily that $T_{\sigma}: \mathcal{S} \rightarrow \mathcal{S}$ is a continuous linear mapping.

The following two results on the basic calculus of SG pseudo-differential operators can be found on page 251 of [7].

THEOREM 1.1. Let $\sigma \in S^{m_{1}, m_{2}}$ and $\tau \in S^{\mu_{1}, \mu_{2}}$. Then $T_{\sigma} T_{\tau}=T_{\lambda}$, where $\lambda \in S^{m_{1}+\mu_{1}, m_{2}+\mu_{2}}$ and

$$
\lambda \sim \sum_{\mu} \frac{(-i)^{|\mu|}}{\mu !}\left(\partial_{\xi}^{\mu} \sigma\right)\left(\partial_{x}^{\mu} \tau\right)
$$

Here, the asymptotic expansion means that for every positive integer $M$, there exists a positive integer $N$ such that

$$
\lambda-\sum_{|\mu|<N} \frac{(-i)^{|\mu|}}{\mu !}\left(\partial_{\xi}^{\mu} \sigma\right)\left(\partial_{x}^{\mu} \tau\right) \in S^{m_{1}+\mu_{1}-M, m_{2}+\mu_{2}-M} .
$$

Theorem 1.2. Let $\sigma \in S^{m_{1}, m_{2}}$. Then the formal adjoint $T_{\sigma}^{*}$ of $T_{\sigma}$ is a pseudo-differential operator $T_{\tau}$, where $\tau \in S^{m_{1}, m_{2}}$ and

$$
\tau \sim \sum_{\mu} \frac{(-i)^{|\mu|}}{\mu !} \partial_{x}^{\mu} \partial_{\xi}^{\mu} \bar{\sigma}
$$

Here, the asymptotic expansion means that for every positive integer $M$, there exists a positive integer $N$ such that

$$
\tau-\sum_{|\mu|<N} \frac{(-i)^{|\mu|}}{\mu !} \partial_{x}^{\mu} \partial_{\xi}^{\mu} \bar{\sigma} \in S^{m_{1}-M, m_{2}-M} .
$$

Using the formal adjoint, we can extend the definition of a pseudodifferential operator from the Schwartz space $\mathcal{S}$ to the space $\mathcal{S}^{\prime}$ of all tempered distributions. Indeed, let $\sigma \in S^{m_{1}, m_{2}}$. Then for all $u$ in $\mathcal{S}^{\prime}$, we define the linear functional $T_{\sigma}: \mathcal{S} \rightarrow \mathbb{C}$ by

$$
\left(T_{\sigma} u\right)(\varphi)=u\left(\overline{T_{\sigma}^{*} \bar{\varphi}}\right), \quad \varphi \in \mathcal{S} .
$$

It is easy to check that $T_{\sigma}$ maps $\mathcal{S}^{\prime}$ into $\mathcal{S}^{\prime}$ continuously. In fact, we have the following theorem.

Theorem 1.3. Let $\sigma \in S^{0,0}$. Then $T_{\sigma}: L^{p}\left(\mathbb{R}^{n}\right) \rightarrow L^{p}\left(\mathbb{R}^{n}\right)$ is a bounded linear operator for $1<p<\infty$. 
Theorem 1.3 follows from Theorem 10.7 in [19] and the fact that every symbol in $S^{0,0}$ is in $S^{0}$.

Let $\sigma \in S^{m_{1}, m_{2}},-\infty<m_{1}, m_{2}<\infty$. Then $\sigma$ is said to be elliptic if there exist positive constants $C$ and $R$ such that

$$
|\sigma(x, \xi)| \geq C\langle x\rangle^{m_{2}}\langle\xi\rangle^{m_{1}}, \quad|x|^{2}+|\xi|^{2} \geq R .
$$

Theorem 1.4. Let $\sigma \in S^{m_{1}, m_{2}},-\infty<m_{1}, m_{2}<\infty$, be elliptic. Then there exists a symbol $\tau$ in $S^{-m_{1},-m_{2}}$ such that

$$
T_{\tau} T_{\sigma}=I+R \quad \text { and } \quad T_{\sigma} T_{\tau}=I+S,
$$

where $R$ and $S$ are infinitely smoothing in the sense that they are $S G$ pseudodifferential operators with symbols in $\bigcap_{k_{1}, k_{2} \in \mathbb{R}} S^{k_{1}, k_{2}}$.

The SG pseudo-differential operator $T_{\tau}$ in Theorem 1.4 is known as a parametrix of $T_{\sigma}$.

The aim of this paper is to investigate the spectral theory of SG pseudodifferential operators with symbols in $S^{m_{1}, m_{2}}, m_{1}, m_{2}>0$, on $L^{p}\left(\mathbb{R}^{n}\right)$, $1<p<\infty$, in the context of minimal and maximal operators, the domains of elliptic SG pseudo-differential operators, Fredholm operators and essential spectra. Results on essential spectra of bounded SG pseudo-differential operators of orders 0,0 on $L^{p}\left(\mathbb{R}^{n}\right)$ are also given. An essential ingredient in the spectral theory is the family of $L^{p}$-Sobolev spaces of orders $s_{1}, s_{2}$, $1<p<\infty,-\infty<s_{1}, s_{2}<\infty$, which we introduce in Section 2. In Section 3, we present the minimal and maximal operators of SG pseudo-differential operators with symbols in $S^{m_{1}, m_{2}}, m_{1}, m_{2}>0$, and show that they are equal for elliptic SG pseudo-differential operators. The main tool is an analogue of the Agmon-Douglis-Nirenberg inequalities for elliptic SG pseudodifferential operators, which we also establish in Section 3. Section 4 is devoted to Fredholmness and essential spectra of elliptic SG pseudo-differential operators with positive orders and bounded SG pseudo-differential operators with orders 0,0 .

The spectral theory for another class of elliptic pseudo-differential operators on $L^{p}\left(\mathbb{R}^{n}\right), 1<p<\infty$, can be found in [20].

2. Sobolev spaces. For $s_{1}, s_{2} \in(-\infty, \infty)$, we let $J_{s_{1}, s_{2}}$ be the Bessel potential of orders $s_{1}, s_{2}$ defined by

$$
J_{s_{1}, s_{2}}=T_{\sigma_{s_{1}, s_{2}}},
$$

where

$$
\sigma_{s_{1}, s_{2}}(x, \xi)=\langle x\rangle^{-s_{2}}\langle\xi\rangle^{-s_{1}}, \quad x, \xi \in \mathbb{R}^{n} .
$$

Obviously, $\sigma_{s_{1}, s_{2}} \in S^{-s_{1},-s_{2}}$. It can be shown easily that the mapping $J_{s_{1}, s_{2}}$ : $\mathcal{S}^{\prime} \rightarrow \mathcal{S}^{\prime}$ is a bijection and

$$
J_{s_{1}, s_{2}}^{-1}=J_{-s_{1}, 0} J_{0,-s_{2}}
$$


and hence, by Theorem 1.1, $J_{s_{1}, s_{2}}^{-1}$ is an SG pseudo-differential operator of orders $-s_{1},-s_{2}$.

For $1<p<\infty$ and $-\infty<s_{1}, s_{2}<\infty$, we define the $L^{p}$-Sobolev space $H^{s_{1}, s_{2}, p}$ of orders $s_{1}, s_{2}$ by

$$
H^{s_{1}, s_{2}, p}=\left\{u \in \mathcal{S}^{\prime}: J_{-s_{1},-s_{2}} u \in L^{p}\left(\mathbb{R}^{n}\right)\right\} .
$$

Then $H^{s_{1}, s_{2}, p}$ is a Banach space in which the norm \|\|$_{s_{1}, s_{2}, p}$ is given by

$$
\|u\|_{s_{1}, s_{2}, p}=\left\|J_{-s_{1},-s_{2}} u\right\|_{L^{p}\left(\mathbb{R}^{n}\right)}, \quad u \in H^{s_{1}, s_{2}, p},
$$

where \|\|$_{L^{p}\left(\mathbb{R}^{n}\right)}$ is the norm in $L^{p}\left(\mathbb{R}^{n}\right)$. Obviously,

$$
H^{0,0, p}=L^{p}\left(\mathbb{R}^{n}\right) .
$$

We also have the following simple proposition.

Proposition 2.1. For $1<p<\infty$ and $-\infty<s_{1}, s_{2}<\infty, J_{-s_{1},-s_{2}}$ : $H^{s_{1}, s_{2}, p} \rightarrow L^{p}\left(\mathbb{R}^{n}\right)$ is a surjective isometry.

Proof. Since

$$
\left\|J_{-s_{1},-s_{2}} u\right\|_{L^{p}\left(\mathbb{R}^{n}\right)}=\|u\|_{s_{1}, s_{2}, p}, \quad u \in H^{s_{1}, s_{2}, p},
$$

it follows that $J_{-s_{1},-s_{2}}: H^{s_{1}, s_{2}, p} \rightarrow L^{p}\left(\mathbb{R}^{n}\right)$ is an isometry. For every $v$ in $L^{p}\left(\mathbb{R}^{n}\right)$, let $u=J_{-s_{1},-s_{2}}^{-1} v$. Then $J_{-s_{1},-s_{2}} u=v \in L^{p}\left(\mathbb{R}^{n}\right)$, and hence $u \in H^{s_{1}, s_{2}, p}$ and the surjectivity is established.

We can now extend the $L^{p}$-boundedness result in Theorem 1.3 from symbols in $S^{0,0}$ to symbols in $S^{m_{1}, m_{2}},-\infty<m_{1}, m_{2}<\infty$.

Theorem 2.2. Let $\sigma \in S^{m_{1}, m_{2}},-\infty<m_{1}, m_{2}<\infty$. Then for $1<p$ $<\infty$ and $-\infty<s_{1}, s_{2}<\infty, T_{\sigma}: H^{s_{1}, s_{2}, p} \rightarrow H^{s_{1}-m_{1}, s_{2}-m_{2}, p}$ is a bounded linear operator.

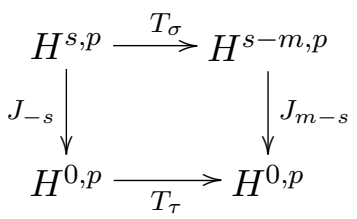

Fig. 1. The vector notation for subscripts and superscripts is used. Precisely, $s=\left(s_{1}, s_{2}\right)$, $m=\left(m_{1}, m_{2}\right)$ and $0=(0,0)$.

Proof. We factorize the pseudo-differential operator $T_{\sigma}$ as in Figure 1 and get

$$
T_{\sigma}=J_{m_{1}-s_{1}, m_{2}-s_{2}}^{-1} T_{\tau} J_{-s_{1},-s_{2}}, \quad \text { where } \quad T_{\tau}=J_{m_{1}-s_{1}, m_{2}-s_{2}} T_{\sigma} J_{-s_{1},-s_{2}}^{-1} .
$$

By Theorem 1.1 and (2.1), we see that $T_{\tau}$ is an SG pseudo-differential operator with symbol in $S^{0,0}$. Hence, by Theorem $1.3, T_{\tau}: L^{p}\left(\mathbb{R}^{n}\right) \rightarrow L^{p}\left(\mathbb{R}^{n}\right)$ is a bounded linear operator, and this completes the proof of the theorem. 
The following Sobolev embedding theorem is the $L^{p}$-analogue of the one in $[5]$.

TheOREM 2.3. Let $s_{1}, s_{2}, t_{1}, t_{2} \in(-\infty, \infty)$ be such that $s_{1} \leq t_{1}$ and $s_{2} \leq t_{2}$. Then $H^{t_{1}, t_{2}, p} \subseteq H^{s_{1}, s_{2}, p}$ and the inclusion $i: H^{t_{1}, t_{2}, p} \hookrightarrow H^{s_{1}, s_{2}, p}$ is a bounded linear operator.

We need a lemma.

Lemma 2.4. Let $s_{1}, s_{2} \geq 0$. Then $H^{s_{1}, s_{2}, p} \subseteq L^{p}\left(\mathbb{R}^{n}\right)$ and

$$
\|u\|_{L^{p}\left(\mathbb{R}^{n}\right)} \leq\|u\|_{s_{1}, s_{2}, p}, \quad u \in H^{s_{1}, s_{2}, p} .
$$

Proof. Let $u \in H^{s_{1}, s_{2}, p}$. Then $J_{-s_{1},-s_{2}} u \in L^{p}\left(\mathbb{R}^{n}\right)$. So,

$$
\langle\rangle^{s_{1}} J_{-s_{2}} u \in L^{p}\left(\mathbb{R}^{n}\right),
$$

where $J_{-s_{2}}$ is the classical pseudo-differential operator with symbol $\sigma_{-s_{2}}$ given by

$$
\sigma_{-s_{2}}(\xi)=\langle\xi\rangle^{s_{2}}, \quad \xi \in \mathbb{R}^{n} .
$$

Therefore $J_{-s_{2}} u \in L^{p}\left(\mathbb{R}^{n}\right)$, which is the same as saying that $u$ is the classical $L^{p}$-Sobolev space $H^{s_{2}, p}$ studied in Chapter 11 of [19]. By Theorem 11.5 in [19], $u \in L^{p}\left(\mathbb{R}^{n}\right)$ and

$$
\|u\|_{L^{p}\left(\mathbb{R}^{n}\right)} \leq\|u\|_{s_{2}, p} \leq\|u\|_{s_{1}, s_{2}, p},
$$

where \|\|$_{s_{2}, p}$ is the norm in $H^{s_{2}, p}$.

Proof of Theorem 2.3. We first suppose that $s_{1} \leq t_{1}$ and $s_{2} \leq t_{2}$. By Lemma 2.4,

$$
\|u\|_{s_{1}, s_{2}, p}=\left\|J_{-s_{1},-s_{2}} u\right\|_{L^{p}\left(\mathbb{R}^{n}\right)} \leq\left\|J_{-s_{1},-s_{2}} u\right\|_{t_{1}-s_{1}, t_{2}-s_{2}, p}, \quad u \in H^{t_{1}, t_{2}, p} .
$$

By Theorem 2.2, there exists a positive constant $C$ such that

$$
\left\|J_{-s_{1},-s_{2}} u\right\|_{t_{1}-s_{1}, t_{2}-s_{2}, p} \leq C\|u\|_{t_{1}, t_{2}, p}, \quad u \in H^{t_{1}, t_{2}, p},
$$

and this completes the proof of the theorem.

Theorem 2.5. Let $s_{1}, s_{2}, t_{1}, t_{2} \in(-\infty, \infty)$ be such that $s_{1}<t_{1}$ and $s_{2}<t_{2}$. Then the inclusion $i: H^{t_{1}, t_{2}, p} \hookrightarrow H^{s_{1}, s_{2}, p}$ is a compact operator.

To prove Theorem 2.5, we recall pseudo-differential operators with symbols first introduced by Grushin [8]. Let $m \in(-\infty, \infty)$. Then we let $S_{0}^{m}$ be the set of all functions $\sigma$ in $C^{\infty}\left(\mathbb{R}^{n} \times \mathbb{R}^{n}\right)$ such that for all multi-indices $\alpha$ and $\beta$, there exists a bounded function $C_{\alpha, \beta}$ on $\mathbb{R}^{n}$ for which

$$
\left|\left(D_{x}^{\alpha} D_{\xi}^{\beta} \sigma\right)(x, \xi)\right| \leq C_{\alpha, \beta}(x)(1+|\xi|)^{m-|\beta|}, \quad x, \xi \in \mathbb{R}^{n},
$$

and

$$
\lim _{|x| \rightarrow \infty} C_{\alpha, \beta}(x)=0
$$


for $|\alpha| \neq 0$. For $\sigma \in S_{0}^{m}$, the pseudo-differential operator $T_{\sigma}$ is defined as in (1.1). Then we have the following theorem proved in $[17,18]$. The $L^{2}$-version of the theorem is in [8].

Theorem 2.6. Let $\sigma \in S_{0}^{m}, m \in(-\infty, \infty)$, be such that

$$
\lim _{|x| \rightarrow \infty} C_{\alpha, \beta}(x)=0
$$

for all multi-indices $\alpha$ and $\beta$. Then for every positive number $\varepsilon, T_{\sigma}: H^{s+m, p}$ $\rightarrow H^{s-\varepsilon, p}$ is a compact operator for $-\infty<s<\infty$ and $1<p<\infty$.

We need the following simple consequence of Theorem 2.6.

Corollary 2.7. For every positive number $\varepsilon, J_{\varepsilon, \varepsilon}: L^{p}\left(\mathbb{R}^{n}\right) \rightarrow L^{p}\left(\mathbb{R}^{n}\right)$ is a compact operator for $1<p<\infty$.

Proof of Theorem 2.5. Let $\varepsilon$ be a positive number such that

$$
t_{1}-s_{1}-\varepsilon>0, \quad t_{2}-s_{2}-\varepsilon>0 .
$$

Since $J_{\varepsilon, \varepsilon}^{-1} J_{-s_{1},-s_{2}}$ is an SG pseudo-differential operator of orders $s_{1}+\varepsilon, s_{2}+\varepsilon$, it follows that the composition $J_{\varepsilon, \varepsilon} i J_{\varepsilon, \varepsilon}^{-1} J_{-s_{1},-s_{2}}$ of the mappings

$$
\begin{aligned}
J_{\varepsilon, \varepsilon}^{-1} J_{-s_{1},-s_{2}} & : H^{t_{1}, t_{2}, p} \rightarrow H^{t_{1}-s_{1}-\varepsilon, t_{2}-s_{2}-\varepsilon, p}, \\
i & : H^{t_{1}-s_{1}-\varepsilon, t_{2}-s_{2}-\varepsilon, p} \hookrightarrow L^{p}\left(\mathbb{R}^{n}\right), \\
J_{\varepsilon, \varepsilon} & : L^{p}\left(\mathbb{R}^{n}\right) \rightarrow L^{p}\left(\mathbb{R}^{n}\right)
\end{aligned}
$$

is compact since $J_{\varepsilon, \varepsilon}^{-1} J_{-s_{1},-s_{2}}: H^{t_{1}, t_{2}, p} \rightarrow H^{t_{1}-s_{1}-\varepsilon, t_{2}-s_{2}-\varepsilon, p}$ is a bounded linear operator by Theorem 2.3 and $J_{\varepsilon, \varepsilon}: L^{p}\left(\mathbb{R}^{n}\right) \rightarrow L^{p}\left(\mathbb{R}^{n}\right)$ is a compact operator by Corollary 2.7. Thus, the linear operator

$$
H^{t_{1}, t_{2}, p} \ni u \mapsto J_{\varepsilon, \varepsilon} i J_{\varepsilon, \varepsilon}^{-1} J_{-s_{1},-s_{2}} u=J_{-s_{1},-s_{2}} u \in L^{p}\left(\mathbb{R}^{n}\right)
$$

is compact, and this completes the proof.

3. Minimal and maximal operators. Let $\sigma \in S^{m_{1}, m_{2}}, m_{1}, m_{2}>0$. Then we can consider $T_{\sigma}$ to be a linear operator from $L^{p}\left(\mathbb{R}^{n}\right)$ into $L^{p}\left(\mathbb{R}^{n}\right)$ with dense domain $\mathcal{S}$ and we can easily prove that it is closable. Thus, the minimal operator $T_{\sigma, 0}$ of $T_{\sigma}$ exists. In fact, the domain $\mathcal{D}\left(T_{\sigma, 0}\right)$ of $T_{\sigma, 0}$ consists of all functions $u$ in $L^{p}\left(\mathbb{R}^{n}\right)$ for which there exists a sequence $\left\{\varphi_{k}\right\}_{k=1}^{\infty}$ in $\mathcal{S}$ such that $\varphi_{k} \rightarrow u$ in $L^{p}\left(\mathbb{R}^{n}\right)$ and $T_{\sigma} \varphi_{k} \rightarrow f$ for some $f$ in $L^{p}\left(\mathbb{R}^{n}\right)$ as $k \rightarrow \infty$. Moreover, if $u \in \mathcal{D}\left(T_{\sigma, 0}\right)$, then it can be shown that the limit $f$ does not depend on the choice of the sequence $\left\{\varphi_{k}\right\}_{k=1}^{\infty}$ in $\mathcal{S}$ and so we can define $T_{\sigma, 0} u$ to be $f$.

Let $u$ and $f$ be functions in $L^{p}\left(\mathbb{R}^{n}\right)$. Then we say that $u \in \mathcal{D}\left(T_{\sigma, 1}\right)$ and $T_{\sigma, 1} u=f$ if and only if

$$
\left(u, T_{\sigma}^{*} \varphi\right)=(f, \varphi), \quad \varphi \in \mathcal{S},
$$


where

$$
(u, v)=\int_{\mathbb{R}^{n}} u(x) \overline{v(x)} d x
$$

for all measurable functions $u$ and $v$ on $\mathbb{R}^{n}$, provided that the integral exists. It can be proved that $T_{\sigma, 1}$ is a closed linear operator from $L^{p}\left(\mathbb{R}^{n}\right)$ into $L^{p}\left(\mathbb{R}^{n}\right)$ with domain $\mathcal{D}\left(T_{\sigma, 1}\right)$ and $\mathcal{S} \subset \mathcal{D}\left(T_{\sigma, 1}\right)$. In fact, we can also prove that $\mathcal{S}$ is contained in the domain $\mathcal{D}\left(T_{\sigma, 1}^{t}\right)$ of the true adjoint $T_{\sigma, 1}^{t}$ of $T_{\sigma, 1}$. Furthermore, $T_{\sigma, 1} u=T_{\sigma} u$ for all $u$ in $\mathcal{D}\left(T_{\sigma, 1}\right)$.

It is a simple fact that $T_{\sigma, 1}$ is an extension of $T_{\sigma, 0}$. Thus, $\mathcal{D}\left(T_{\sigma, 1}^{t}\right) \subseteq$ $\mathcal{D}\left(T_{\sigma, 0}^{t}\right)$ and hence $\mathcal{S} \subset \mathcal{D}\left(T_{\sigma, 0}^{t}\right)$. In fact, $T_{\sigma, 1}$ is the largest closed extension of $T_{\sigma}$ in the sense that if $B$ is any closed extension of $T_{\sigma}$ such that $\mathcal{S} \subset \mathcal{D}\left(B^{t}\right)$, then $T_{\sigma, 1}$ is an extension of $B$. So, $T_{\sigma, 1}$ is called the maximal operator of $T_{\sigma}$.

The main results that we want to prove in this section are given by the following theorems.

Theorem 3.1. If $\sigma \in S^{m_{1}, m_{2}}, m_{1}, m_{2}>0$, is elliptic, then $T_{\sigma, 0}=T_{\sigma, 1}$.

Theorem 3.2. If $\sigma \in S^{m_{1}, m_{2}}, m_{1}, m_{2}>0$, is elliptic, then $\mathcal{D}\left(T_{\sigma, 0}\right)=$ $H^{m_{1}, m_{2}, p}$.

We give a proof of Theorem 3.2 based on the following result that contains an analogue of the Agmon-Douglis-Nirenberg inequalities in [1] for SG pseudo-differential operators.

Theorem 3.3. Let $\sigma \in S^{m_{1}, m_{2}}, m_{1}, m_{2}>0$, be elliptic. Then there exist positive constants $C_{1}$ and $C_{2}$ such that

$C_{1}\|u\|_{m_{1}, m_{2}, p} \leq\left\|T_{\sigma} u\right\|_{L^{p}\left(\mathbb{R}^{n}\right)}+\|u\|_{L^{p}\left(\mathbb{R}^{n}\right)} \leq C_{2}\|u\|_{m_{1}, m_{2}, p}, \quad u \in H^{m_{1}, m_{2}, p}$.

Proof. By Theorem 2.2 on boundedness of SG pseudo-differential operators between Sobolev spaces and Theorem 2.3 on the boundedness of the Sobolev embedding, there exists a positive constant $C^{\prime}$ such that

$$
\left\|T_{\sigma} u\right\|_{L^{p}\left(\mathbb{R}^{n}\right)}+\|u\|_{L^{p}\left(\mathbb{R}^{n}\right)} \leq C^{\prime}\|u\|_{m_{1}, m_{2}, p}, \quad u \in H^{m_{1}, m_{2}, p} .
$$

Since $\sigma$ is elliptic, Theorem 1.4 ensures that there exists a symbol $\tau$ in $S^{-m_{1},-m_{2}}$ such that

$$
u=T_{\tau} T_{\sigma} u-R u, \quad u \in H^{m_{1}, m_{2}, p},
$$

where $R$ is an SG pseudo-differential operator with symbol in $\bigcap_{k_{1}, k_{2} \in \mathbb{R}} S^{k_{1}, k_{2}}$. So, by Theorem 2.2 again, there exists a positive constant $C$ such that

$$
\|u\|_{m_{1}, m_{2}, p} \leq C\left(\left\|T_{\sigma} u\right\|_{L^{p}\left(\mathbb{R}^{n}\right)}+\|u\|_{L^{p}\left(\mathbb{R}^{n}\right)}\right), \quad u \in H^{m_{1}, m_{2}, p} .
$$

This completes the proof.

We also need the following result.

Proposition 3.4. For $-\infty<s_{1}, s_{2}<\infty$ and $1<p<\infty, \mathcal{S}$ is dense in $H^{s_{1}, s_{2}, p}$. 
Proof. Let $u \in H^{s_{1}, s_{2}, p}$. Then $J_{-s_{1},-s_{2}} u \in L^{p}\left(\mathbb{R}^{n}\right)$. Since $\mathcal{S}$ is dense in $L^{p}\left(\mathbb{R}^{n}\right)$, we can find a sequence $\left\{\varphi_{k}\right\}_{k=1}^{\infty}$ in $\mathcal{S}$ such that $\varphi_{k} \rightarrow u$ in $H^{s_{1}, s_{2}, p}$ as $k \rightarrow \infty$. For $k=1,2, \ldots$, let $\psi_{k}=J_{-s_{1},-s_{2}}^{-1} \varphi_{k}$. Then $\psi_{k} \in \mathcal{S}, k=1,2, \ldots$, and

$\left\|\psi_{k}-u\right\|_{s_{1}, s_{2}, p}=\left\|J_{-s_{1},-s_{2}} \psi_{k}-J_{-s_{1},-s_{2}} u\right\|_{L^{p}\left(\mathbb{R}^{n}\right)}=\left\|\varphi_{k}-J_{-s_{1},-s_{2}} u\right\|_{L^{p}\left(\mathbb{R}^{n}\right)} \rightarrow 0$ as $k \rightarrow \infty$, and this completes the proof of the theorem.

Proof of Theorem 3.2. Let $u \in H^{m_{1}, m_{2}, p}$. By the density of $\mathcal{S}$ in $H^{m_{1}, m_{2}, p}$, we can find a sequence $\left\{\varphi_{k}\right\}_{k=1}^{\infty}$ in $\mathcal{S}$ such that $\varphi_{k} \rightarrow u$ in $H^{m_{1}, m_{2}, p}$ as $k \rightarrow \infty$. By the second of the Agmon-Douglis-Nirenberg inequalities in Theorem 3.3, we see that $\left\{T_{\sigma} \varphi_{k}\right\}_{k=1}^{\infty}$ and $\left\{\varphi_{k}\right\}_{k=1}^{\infty}$ are Cauchy sequences in $L^{p}\left(\mathbb{R}^{n}\right)$. Hence $\varphi_{k} \rightarrow u$ and $T_{\sigma} \varphi_{k} \rightarrow f$ for some $u$ and $f$ in $L^{p}\left(\mathbb{R}^{n}\right)$ as $k \rightarrow \infty$. Hence $u \in \mathcal{D}\left(T_{\sigma, 0}\right)$ and $T_{\sigma, 0} u=f$. Now, let $u \in \mathcal{D}\left(T_{\sigma, 0}\right)$. Then there exists a sequence $\left\{\varphi_{k}\right\}_{k=1}^{\infty}$ in $\mathcal{S}$ such that $\varphi_{k} \rightarrow u$ in $L^{p}\left(\mathbb{R}^{n}\right)$ and $T_{\sigma} \varphi_{k} \rightarrow f$ for some $f$ in $L^{p}\left(\mathbb{R}^{n}\right)$ as $k \rightarrow \infty$. So, $\{\varphi\}_{k=1}^{\infty}$ and $\left\{T_{\sigma} \varphi_{k}\right\}_{k=1}^{\infty}$ are Cauchy sequences in $L^{p}\left(\mathbb{R}^{n}\right)$. By the first of the Agmon-Douglis-Nirenberg inequalities in Theorem 3.3, we see that $\{\varphi\}_{k=1}^{\infty}$ is a Cauchy sequence in $H^{m_{1}, m_{2}, p}$. Since $H^{m_{1}, m_{2}, p}$ is complete, it follows that $\varphi_{k} \rightarrow v$ in $H^{m_{1}, m_{2}, p}$ for some $v$ in $H^{m_{1}, m_{2}, p}$ as $k \rightarrow \infty$. So, by Theorem 2.3 on the boundedness of the Sobolev embedding, $\varphi_{k} \rightarrow v$ in $L^{p}\left(\mathbb{R}^{n}\right)$. Hence $u=v$ and $u \in H^{m_{1}, m_{2}, p}$.

Proof of Theorem 3.1. Since $T_{\sigma, 1}$ is an extension of $T_{\sigma, 0}$ and $\mathcal{D}\left(T_{\sigma, 0}\right)=$ $H^{m_{1}, m_{2}, p}$, it is enough to prove that $\mathcal{D}\left(T_{\sigma, 1}\right) \subseteq H^{m_{1}, m_{2}, p}$. Let $u \in \mathcal{D}\left(T_{\sigma, 1}\right)$. Since $\sigma$ is elliptic, it follows from Theorem 1.4 that there exists a symbol $\tau$ in $S^{-m_{1},-m_{2}}$ such that

$$
u=T_{\tau} T_{\sigma} u-R u,
$$

where $R$ is an SG pseudo-differential operator with symbol in $\bigcap_{k_{1}, k_{2} \in \mathbb{R}} S^{k_{1}, k_{2}}$. Since

$$
T_{\sigma} u=T_{\sigma, 1} u \in L^{p}\left(\mathbb{R}^{n}\right),
$$

it follows from the boundedness of SG pseudo-differential operators between Sobolev spaces in Theorem 2.2 that $u \in H^{m_{1}, m_{2}, p}$. So, $\mathcal{D}\left(T_{\sigma, 1}\right) \subseteq H^{m_{1}, m_{2}, p}$, as asserted.

4. Fredholm SG pseudo-differential operators. Let us first recall that a closed linear operator $A$ from a complex Banach space $X$ into a complex Banach space $Y$ with dense domain $\mathcal{D}(A)$ is said to be Fredholm if the range $R(A)$ of $A$ is a closed subspace of $Y$, and the null space $N(A)$ of $A$ and the null space $N\left(A^{t}\right)$ of the true adjoint $A^{t}$ of $A$ are finite-dimensional. For a Fredholm operator $A$, the index $i(A)$ of $A$ is defined by

$$
i(A)=\operatorname{dim} N(A)-\operatorname{dim} N\left(A^{t}\right) .
$$


The following criterion for a closed linear operator is usually attributed to Atkinson [2].

TheOREM 4.1. Let $A$ be a closed linear operator from a complex Banach space $X$ into a complex Banach space $Y$ with dense domain $\mathcal{D}(A)$. Then $A$ is Fredholm if and only if we can find a bounded linear operator $B: Y \rightarrow X$, a compact operator $K_{1}: X \rightarrow X$ and a compact operator $K_{2}: Y \rightarrow Y$ such that $B A=I+K_{1}$ on $\mathcal{D}(A)$ and $A B=I+K_{2}$ on $Y$.

Let $X$ be a complex Banach space and let $A$ be a closed linear operator from $X$ into $X$ with dense domain $\mathcal{D}(A)$. Then the spectrum $\Sigma(A)$ of $A$ is defined in the usual way, i.e.,

$$
\Sigma(A)=\mathbb{C} \backslash \varrho(A),
$$

where $\varrho(A)$ is the resolvent set of $A$ given by

$$
\varrho(A)=\{\lambda \in \mathbb{C}: A-\lambda I \text { is bijective }\}
$$

and $I$ is the identity operator on $X$. The essential spectrum $\Sigma_{\mathrm{w}}(A)$ of $A$ in the sense of Wolf [16] is defined by

$$
\Sigma_{\mathrm{w}}(A)=\mathbb{C} \backslash \Phi_{\mathrm{w}}(A), \quad \text { where } \quad \Phi_{\mathrm{w}}(A)=\{\lambda \in \mathbb{C}: A-\lambda I \text { is Fredholm }\} .
$$

An important fact is that $i(A-\lambda I)$ is a constant for all $\lambda$ in a connected component of $\Phi_{\mathrm{w}}(A)$. The essential spectrum $\Sigma_{\mathrm{s}}(A)$ of $A$ in the sense of Schechter $[13]$ is defined by

$$
\Sigma_{\mathrm{s}}(A)=\mathbb{C} \backslash \Phi_{\mathrm{s}}(A), \quad \text { where } \quad \Phi_{\mathrm{s}}(A)=\left\{\lambda \in \Phi_{\mathrm{w}}(A): i(A-\lambda I)=0\right\} .
$$

All the results on Fredholm operators hitherto described can be found in the books [14], [15] by Schechter.

The first main result in this section is the following theorem.

TheOREM 4.2. Let $\sigma \in S^{m_{1}, m_{2}}, m_{1}, m_{2}>0$, be elliptic. Then for $1<$ $p<\infty, T_{\sigma, 0}$ is a Fredholm operator on $L^{p}\left(\mathbb{R}^{n}\right)$ with domain $H^{m_{1}, m_{2}, p}$. Furthermore, if $\sigma \in S^{0,0}$ is elliptic, then the bounded linear operator $T_{\sigma}$ : $L^{p}\left(\mathbb{R}^{n}\right) \rightarrow L^{p}\left(\mathbb{R}^{n}\right)$ is Fredholm.

Proof. Since $\sigma$ is elliptic, it follows from Theorem 1.4 that there exists a symbol $\tau$ in $S^{-m_{1},-m_{2}, p}$ such that

$$
T_{\tau} T_{\sigma}=I+R \quad \text { and } \quad T_{\sigma} T_{\tau}=I+S,
$$

where $R$ and $S$ are infinitely smoothing in the sense that they are SG pseudodifferential operators with symbols in $\bigcap_{k_{1}, k_{2} \in \mathbb{R}} S^{k_{1}, k_{2}}$. So, for all positive numbers $t_{1}$ and $t_{2}$, the linear operator $R: L^{p}\left(\mathbb{R}^{n}\right) \rightarrow L^{p}\left(\mathbb{R}^{n}\right)$ is the same as the composition of the linear operators $R: L^{p}\left(\mathbb{R}^{n}\right) \rightarrow H^{t_{1}, t_{2}, p}$ and $i$ : $H^{t_{1}, t_{2}, p} \hookrightarrow L^{p}\left(\mathbb{R}^{n}\right)$. Since $R: L^{p}\left(\mathbb{R}^{n}\right) \rightarrow H^{t_{1}, t_{2}, p}$ is bounded by Theorem 2.2 and $i: H^{t_{1}, t_{2}, p} \hookrightarrow L^{p}\left(\mathbb{R}^{n}\right)$ is compact by Theorem 2.5 , it follows that $R: L^{p}\left(\mathbb{R}^{n}\right) \rightarrow L^{p}\left(\mathbb{R}^{n}\right)$ is compact. Similarly, $S: L^{p}\left(\mathbb{R}^{n}\right) \rightarrow L^{p}\left(\mathbb{R}^{n}\right)$ is 
compact. So, by Theorem 4.1, $T_{\sigma, 0}$ is a compact, as asserted. The proof for the Fredholmness of $T_{\sigma}: L^{p}\left(\mathbb{R}^{n}\right) \rightarrow L^{p}\left(\mathbb{R}^{n}\right)$ when $\sigma \in S^{0,0}$ is the same.

The following theorem gives the essential spectrum in the sense of Wolf of an elliptic SG pseudo-differential operator.

Theorem 4.3. Let $\sigma \in S^{m_{1}, m_{2}}, m_{1}, m_{2}>0$, be elliptic. Then

$$
\Sigma_{\mathrm{w}}\left(T_{\sigma, 0}\right)=\emptyset .
$$

Proof. We only need to prove that $\sigma-\lambda$ is elliptic for all $\lambda$ in $\mathbb{C}$, for then by Theorem 4.2, $T_{\sigma, 0}-\lambda I$ is a Fredholm operator on $L^{p}\left(\mathbb{R}^{n}\right)$ with domain $H^{m_{1}, m_{2}, p}$. By the ellipticity of $\sigma$, there exist positive constants $C$ and $R$ such that

$$
|\sigma(x, \xi)-\lambda| \geq C\langle x\rangle^{m_{2}}\langle\xi\rangle^{m_{1}}=\langle x\rangle^{m_{2}}\langle\xi\rangle^{m_{1}}\left(C-\frac{|\lambda|}{\langle x\rangle^{m_{2}}\langle\xi\rangle^{m_{1}}}\right)
$$

whenever $|x|^{2}+|\xi|^{2} \geq R$. Since $\langle x\rangle^{m_{2}}\langle\xi\rangle^{m_{1}} \rightarrow \infty$ as $|x|^{2}+|\xi|^{2} \rightarrow \infty$, it follows that there exists another positive constant $R^{\prime}$ such that

$$
|\sigma(x, \xi)-\lambda| \geq \frac{C}{2}\langle x\rangle^{m_{2}}\langle\xi\rangle^{m_{1}}
$$

whenever $|x|^{2}+|\xi|^{2} \geq R^{\prime}$. Thus, $\sigma-\lambda$ is elliptic.

The proof of Theorem 4.3 depends on the hypothesis that $\sigma$ is a symbol with positive orders. If $\sigma \in S^{0,0}$, then by Theorem $2.2, T_{\sigma}: L^{p}\left(\mathbb{R}^{n}\right) \rightarrow$ $L^{p}\left(\mathbb{R}^{n}\right)$ is a bounded linear operator and we have the following result on the essential spectra of $T_{\sigma}$.

TheOREM 4.4. Let $\sigma \in S^{0,0}$. Then

$$
\Sigma_{\mathrm{s}}\left(T_{\sigma}\right) \subseteq\left\{\lambda \in \mathbb{C}:|\lambda| \leq L_{\mathrm{s}}\right\}, \quad \Sigma_{\mathrm{w}}\left(T_{\sigma}\right) \subseteq\left\{\lambda \in \mathbb{C}:|\lambda| \geq L_{\mathrm{i}}\right\},
$$

where

$$
L_{\mathrm{i}}=\liminf _{|(x, \xi)| \rightarrow \infty}|\sigma(x, \xi)|, \quad L_{\mathrm{s}}=\limsup _{|(x, \xi)| \rightarrow \infty}|\sigma(x, \xi)| .
$$

REMARK 4.5. A proof for $\Sigma_{\mathrm{s}}\left(T_{\sigma}\right) \subseteq\left\{\lambda \in \mathbb{C}:|\lambda| \leq L_{\mathrm{s}}\right\}$ can be found in [11] by Nicola and Rodino using more advanced techniques. We give a completely elementary proof here.

Proof of Theorem 4.4. Let $\lambda \in \mathbb{C}$ be such that $|\lambda|>L_{\mathrm{s}}$. Let $\varepsilon$ be a positive number such that

$$
|\lambda|-\varepsilon>L_{\mathrm{s}} .
$$

Then there exists a positive number $R$ such that

$$
\sup _{|x|^{2}+|\xi|^{2} \geq R}|\sigma(x, \xi)|<L_{\mathrm{s}}+\varepsilon / 2 .
$$

So, for $|x|^{2}+|\xi|^{2} \geq R$

$$
|\sigma(x, \xi)-\lambda| \geq|\lambda|-|\sigma(x, \xi)|>L_{\mathrm{s}}+\varepsilon-L_{\mathrm{s}}-\varepsilon / 2=\varepsilon / 2 .
$$


Therefore $\sigma-\lambda$ is elliptic and hence $T_{\sigma}-\lambda I: L^{p}\left(\mathbb{R}^{n}\right) \rightarrow L^{p}\left(\mathbb{R}^{n}\right)$ is Fredholm by Theorem 2.2. Thus, $\left\{\lambda \in \mathbb{C}:|\lambda|>L_{\mathrm{s}}\right\} \subseteq \Phi_{\mathrm{w}}\left(T_{\sigma}\right)$, which is the same as

$$
\Sigma_{\mathrm{w}}\left(T_{\sigma}\right) \subseteq\left\{\lambda \in \mathbb{C}:|\lambda| \leq L_{\mathrm{s}}\right\} .
$$

Since $i\left(T_{\sigma}-\lambda I\right)$ is constant for all $\lambda$ in $\left\{\lambda \in \mathbb{C}:|\lambda|>L_{\mathrm{s}}\right\}$ and

$$
\varrho\left(T_{\sigma}\right) \cap\left\{\lambda \in \mathbb{C}:|\lambda|>L_{\mathrm{s}}\right\} \neq \emptyset,
$$

it follows that $i\left(T_{\sigma}-\lambda I\right)=0$ for all $\lambda$ in $\left\{\lambda \in \mathbb{C}:|\lambda|>L_{\mathrm{s}}\right\}$. Thus,

$$
\Sigma_{\mathrm{s}}\left(T_{\sigma}\right) \subseteq\left\{\lambda \in \mathbb{C}:|\lambda| \leq L_{\mathrm{s}}\right\} .
$$

Now, let $\lambda \in \mathbb{C}$ be such that $|\lambda|<\lambda_{i}$. Let $\varepsilon$ be a positive number such that

$$
|\lambda|+\varepsilon<L_{\mathrm{i}} .
$$

Then there exists a positive number $R$ such that

$$
\inf _{|x|^{2}+|\xi|^{2} \geq R}|\sigma(x, \xi)|>L_{\mathrm{i}}-\varepsilon / 2 .
$$

So, for $|x|^{2}+|\xi|^{2} \geq R$,

$$
|\sigma(x, \xi)-\lambda| \geq|\sigma(x, \xi)|-|\lambda|>L_{\mathrm{i}}-\varepsilon / 2-L_{\mathrm{i}}+\varepsilon=\varepsilon / 2 .
$$

Therefore $\sigma-\lambda$ is elliptic and hence $T_{\sigma}-\lambda I: L^{p}\left(\mathbb{R}^{n}\right) \rightarrow L^{p}\left(\mathbb{R}^{n}\right)$ is Fredholm by Theorem 4.2. Thus, $\left\{\lambda \in \mathbb{C}:|\lambda|<L_{\mathrm{i}}\right\} \subseteq \Phi_{\mathrm{w}}\left(T_{\sigma}\right)$, or $\Sigma_{\mathrm{w}}\left(T_{\sigma}\right) \subseteq\{\lambda \in \mathbb{C}$ : $\left.|\lambda| \geq L_{\mathrm{i}}\right\}$.

As a consequence of Theorem 4.4, we have the following spectral alternative for a class of elliptic SG pseudo-differential operators with symbols in $S^{0,0}$.

Theorem 4.6. Let $\sigma \in S^{0,0}$ be such that

$$
\lim _{|(x, \xi)| \rightarrow \infty}|\sigma(x, \xi)|=L>0 .
$$

Then

$$
\Sigma_{\mathrm{w}}\left(T_{\sigma}\right)=\{\lambda \in \mathbb{C}:|\lambda|=L\} \quad \text { or } \quad \Sigma_{\mathrm{s}}\left(T_{\sigma}\right) \subseteq\{\lambda \in \mathbb{C}:|\lambda|=L\} .
$$

Proof. By Theorem 4.4,

$$
\Sigma_{\mathrm{w}}\left(T_{\sigma}\right) \subseteq\{\lambda \in \mathbb{C}:|\lambda|=L\} .
$$

Suppose that $\Sigma_{\mathrm{w}}\left(T_{\sigma}\right) \neq\{\lambda \in \mathbb{C}:|\lambda|=L\}$. Then there exists a complex number $\lambda_{0}$ such that $\left|\lambda_{0}\right|=L$ and $\lambda_{0} \in \Phi_{\mathrm{w}}\left(T_{\sigma}\right)$. So, using the first conclusion in Theorem 4.4, the fact that $\Phi_{\mathrm{w}}\left(T_{\sigma}\right)$ is an open set and that $i\left(T_{\sigma}-\lambda I\right)$ is constant on every connected component of $\Phi_{\mathrm{w}}\left(T_{\sigma}\right)$, we see that $i\left(T_{\sigma}-\lambda I\right)=0$ for all $\lambda$ in $\mathbb{C}$ with $|\lambda| \neq L$. So,

$$
\Sigma_{\mathrm{s}}\left(T_{\sigma}\right) \subseteq\{\lambda \in \mathbb{C}:|\lambda|=L\},
$$

as asserted. 
REMARK 4.7. Suppose that

$$
\Sigma_{\mathrm{w}}\left(T_{\sigma}\right)=\{\lambda \in \mathbb{C}:|\lambda|=L\} .
$$

Then the best that we can say about $\Sigma_{\mathrm{S}}\left(T_{\sigma}\right)$ is given by the first conclusion of Theorem 4.4 to the effect that

$$
\Sigma_{\mathrm{s}}\left(T_{\sigma}\right) \subseteq\{\lambda \in \mathbb{C}:|\lambda| \leq L\} .
$$

To see this by means of an example, let $\sigma$ be the symbol in $S^{0,0}$ given by

$$
\sigma(x, \xi)=L e^{i \arg (x+i \xi)}
$$

for all $x$ and $\xi$ in $\mathbb{R}$ such that $x^{2}+\xi^{2} \geq 1$. Then $T_{\sigma}: L^{2}(\mathbb{R}) \rightarrow L^{2}(\mathbb{R})$ is a Fredholm operator with nonzero index. In other words, for $p=2$, $0 \in \Sigma_{\mathrm{s}}\left(T_{\sigma}\right) \backslash \Sigma_{\mathrm{w}}\left(T_{\sigma}\right)$. So,

$$
\Sigma_{\mathrm{s}}\left(T_{\sigma}\right) \nsubseteq\{\lambda \in \mathbb{C}:|\lambda|=L\} .
$$

See, for instance, Theorem 2.3 in Chapter 5 of the book [9] by Kumano-go for more details.

Acknowledgments. We are grateful to the referee for the very useful comments that have improved the formulation and the presentation of the spectral alternative given in Theorem 4.6 and Remark 4.7.

\section{References}

[1] S. Agmon, A. Douglis and L. Nirenberg, Estimates near the boundary for solutions of elliptic partial differential equations satisfying general boundary conditions I, Comm. Pure Appl. Math. 12 (1959), 623-727.

[2] F. V. Atkinson, The normal solubility of linear equations in normed spaces, Mat. Sb. 28 (70) (1951), 3-14 (in Russian).

[3] P. Boggiatto, E. Buzano and L. Rodino, Global Hypoellipticity and Spectral Theory, Akademie-Verlag, 1996.

[4] M. Cappiello and L. Rodino, SG-pseudo-differential operators and Gelfand-Shilov spaces, Rocky Mountain J. Math. 36 (2006), 1117-1148.

[5] H. O. Cordes, The Technique of Pseudodifferential Operators, Cambridge Univ. Press, 1995.

[6] S. Coriasco and L. Rodino, Cauchy problem for SG-hyperbolic equations with constant multiplicities, Ricerche Mat. 48 (1999), Suppl., 25-43.

[7] Yu. V. Egorov and B.-W. Schulze, Pseudo-Differential Operators, Singularities, Applications, Birkhäuser, 1997.

[8] V. V. Grushin, Pseudo-differential operators on $\mathbb{R}^{n}$ with bounded symbols, Funktsional. Anal. i Prilozhen. 4 (1970), no. 3, 37-50 (in Russian); English transl.: Funct. Anal. Appl. 4 (1970), 202-212.

[9] H. Kumano-go, Pseudo-Differential Operators, MIT Press, 1981.

[10] F. Nicola, K-theory of SG-pseudo-differential algebras, Proc. Amer. Math. Soc. 131 (2003), 2841-2848.

[11] F. Nicola and L. Rodino, SG pseudo-differential operators and weak hyperbolicity, Pliska Stud. Math. Bulgar. 15 (2002), 5-19. 
[12] C. Parenti, Operatori pseudo-differentiali in $\mathbb{R}^{n}$ e applicazioni, Ann. Mat. Pura Appl. 93 (1972), 359-389.

[13] M. Schechter, On the essential spectrum of an arbitrary operator I, J. Math. Anal. Appl. 13 (1966), 205-215.

[14] —, Spectra of Partial Differential Operators, 2nd ed., North-Holland, 1986.

[15] —, Principles of Functional Analysis, 2nd ed., Amer. Math. Soc., 2002.

[16] F. Wolf, On the essential spectrum of partial differential boundary problems, Comm. Pure Appl. Math. 12 (1959), 211-228.

[17] M. W. Wong, Fredholm pseudo-differential operators on weighted Sobolev spaces, Ark. Mat. 21 (1983), 271-282.

[18] —, Spectral theory of pseudo-differential operators, Adv. Appl. Math. 15 (1994), 437-451.

[19] -, An Introduction to Pseudo-Differential Operators, 2nd ed., World Sci., 1999.

[20] - M-elliptic pseudo-differential operators on $L^{p}\left(\mathbb{R}^{n}\right)$, Math. Nachr. 279 (2006), 319-326.

Department of Mathematics and Statistics

York University

4700 Keele Street

Toronto, ON, Canada M3J 1P3

E-mail: adgupta@mathstat.yorku.ca

mwwong@mathstat.yorku.ca

Received August 31, 2007

Revised version April 9, 2008 